\title{
Observation and Interview-based Diurnal Sleepiness Inventory for measurement of sleepiness in older adults
}

This article was published in the following Dove Press journal:

Nature and Science of Sleep

29 September 2017

Number of times this article has been viewed

\author{
Victoria M Pak ${ }^{1,2}$ \\ S-Hakki Onen ${ }^{3,4}$ \\ Nalaka S Gooneratne ${ }^{4}$ \\ Bruno Falissard ${ }^{5}$ \\ Fannie Onen ${ }^{4-6}$ \\ 'Center for Sleep and Circadian \\ Neurobiology, University of \\ Pennsylvania, Philadelphia, PA, ${ }^{2}$ Nell \\ Hodgson Woodruff School of Nursing, \\ Emory University, Atlanta, GA, USA; \\ ${ }^{3} \mathrm{CHU}$ Lyon, Hôpital Edouard Herriot, \\ Geriatric Sleep Medicine Center, \\ Lyon, France; ${ }^{4}$ Division of Geriatric \\ Medicine, Department of Medicine, \\ University of Pennsylvania Perelman \\ School of Medicine, Philadelphia, PA, \\ USA; ${ }^{5} \mathrm{CHU}$ Bichat Claude Bernard, \\ Gériatrie, APHP, Paris, ${ }^{6} \mathrm{CESP}$, INSERM \\ 1018 \& 1178 , Université Paris Sud, \\ Paris, France
}

Introduction: There is no established reference standard for subjective measures of sleepiness in older adults.

Methods: This study compares the Observation and Interview-based Diurnal Sleepiness Inventory (ODSI) with two existing instruments for measurement of sleepiness and daily functioning, the Epworth Sleepiness Scale (ESS) and Functional Outcomes of Sleep Questionnaire (FOSQ). Results: A total of 125 study participants were included in this study and were administered the ODSI, ESS and FOSQ; subjects had a mean age of 70.9 \pm 5.27 years, mean Apnea-Hypopnea Index of $31.9 \pm 27.9$ events/hour and normal cognitive functioning (Mini-Mental State Examination score $>24$ ). The ODSI showed a significant association with the ESS (Spearman's $\rho: 0.67$, $P<0.001$ ) and with the FOSQ (Spearman's $\rho$ : $-0.52, P<0.001)$. The ODSI 1 item (assessing sleepiness in active situations) was borderline significantly correlated with the ESS $(\beta=0.14$; $95 \%$ confidence interval $[\mathrm{CI}],-0.01$ to $0.29 ; P=0.069$ ). ODSI 2 item (sleepiness in passive situations) was correlated with the $\operatorname{ESS}(\beta=1.65 ; 95 \% \mathrm{CI}, 1.32$ to $1.98 ; P<0.001)$. Both ODSI $1(\beta=-0.15 ; 95 \% \mathrm{CI},-0.24$ to $-0.07 ; P<0.001)$ and ODSI $2(\beta=-0.35 ; 95 \% \mathrm{CI},-0.55$ to $0.16 ; P<0.001)$ were significantly correlated with the FOSQ.

Conclusion: The ODSI is a suitable measure of sleepiness and is appropriate for usage in clinical care in older adults.

Keywords: excessive daytime sleepiness, functional status, sleep disorders, questionnaires

\section{Plain language summary}

There is no established reference standard for subjective sleepiness measurement in older adults. The Observation and Interview-based Diurnal Sleepiness Inventory (ODSI) is a suitable measurement of sleepiness for usage in clinical care in older adults. This study compares the ODSI with two existing instruments for measurement of sleepiness and daily functioning.

\section{Introduction}

Excessive daytime sleepiness (EDS) is prevalent among older adults. It affects $\sim 10-30 \%$ of adults aged $>65$ years ${ }^{1-4}$ and is associated with falls, ${ }^{5}$ impaired cognitive function ${ }^{6}$ and mortality. ${ }^{7,8}$ There is evidence that disorders of hypersomnolence continue to be underdiagnosed and consequently undertreated, and there is no established reference standard for subjective measures of sleepiness in older adults. ${ }^{9}$

\section{Current tools}

Most of the currently available tools to assess daytime sleepiness have not been validated in geriatric settings. Examples of existing instruments include the Epworth
Nell Hodgson Woodruff School of

Nursing, Emory University, I520 Clifton

Road NE, Atlanta, GA 30322, USA

Email Victoria.m.pak@emory.edu 
Sleepiness Scale (ESS) ${ }^{10}$ and Functional Outcomes of Sleep Questionnaire (FOSQ). ${ }^{11}$ Current instruments may inquire about sleepiness symptoms in situations that may not apply to all older adults; for example, many older adults no longer drive but sleepiness while driving often is one of the items on the existing assessments. Additional limitations of the ESS include the accuracy of the ESS score being compromised by advanced age and cognitive impairment. ${ }^{12}$ The ESS is intended for independent active people, but older adults may not engage in all of activities identified by the ESS. ${ }^{12}$ Furthermore, Onen et a ${ }^{12}$ determined almost $60 \%$ of subjects, and proxies were not able to answer at least one question on the ESS. Similarly, a limitation of the FOSQ tool is that it may also result in missing items due to the specificity of the questions as pertaining to certain activities that may not apply to older adults.

The purpose of this manuscript is to compare the agreement and response rates of the Observation and Interview-based Diurnal Sleepiness Inventory (ODSI) with two instruments for measurement of sleepiness and daily functioning, the ESS and FOSQ. The ODSI questionnaire has previously been validated using the ESS as the gold standard in a sample of 133 primary care patients, 60 of whom were controls, and demonstrated acceptable validity, internal consistency and test-retest reliability properties. ${ }^{13}$ The ODSI has shown internal consistency and a reliability coefficient (Pearson's $\rho$ ) of 0.70 for its three items. ${ }^{13}$ The estimated sensitivity and specificity were 0.842 and 0.851 with $95 \%$ confidence interval (CI) of 0.624 to 0.945 and 0.761 to 0.911 , respectively. The consistency of summated scale scores during test and retest sessions was high $(r=0.970$, $95 \%$ bootstrap CI [0.898 to 0.991$]) .{ }^{13}$ In a receiver operating characteristic analysis, a cutoff point of 6 on the ODSI is effective for identifying older adults with excessive levels of daytime sleepiness. ${ }^{13}$ Simple screening tools like the ODSI have the potential to improve the accuracy of ascertaining sleepy versus non-sleepy subjects to facilitate the identification of treatable primary sleep disorders among elderly patients.

\section{Methods}

\section{Study subjects}

The proposed study is a cross-sectional study that consisted of survey questionnaires administered to 125 outpatients who attended the Sleep Disorders Clinic at the University of Pennsylvania Health System. Enrolled subjects underwent a polysomnography and were also asked to complete the ODSI, ESS, FOSQ and the Mini-Mental State Examination (MMSE). Sample size estimations were based on prior studies. ${ }^{14,15}$ All subjects provided informed consent prior to filling out the research study survey forms administered by the same study coordinator for whom no training was required to administer this questionnaire as instructions were indicated on the document. The selection criteria included normal cognitive functioning (MMSE score $>24$ ) ${ }^{16}$ and age $>65$ years. Subjects were excluded if they had a history of uncontrolled acute organic disease (i.e., infection, diabetes, metabolic disorders), uncontrolled acute psychiatric disease (i.e., major depression, psychosis, panic attacks) and dementia (Diagnostic and Statistical Manual of Mental Disorders, Fourth Edition [DSM-IV]) or were not able to comprehend English because validated non-English versions of all the study questionnaires were not available at the time of the study. The study was approved by the institutional review board of the University of Pennsylvania.

\section{Instruments}

\section{ESS}

The ESS is a widely used standardized self-report instrument that assesses propensity for sleepiness. ${ }^{10}$ The ESS is a self-administered questionnaire that aims to assess the degree of sleepiness during eight common scenarios. Subjects are asked to rate their chance of dozing during common situations and are asked to rate their chance of dozing in recent times. This is rated on a Likert-type scale ranging from 0 (no chance of dozing) to 3 (high chance of dozing during common situations such as reading or watching TV). Scores are summed with higher scores indicating a greater sleepiness. ESS score correlates with the frequency of apneas and is a validated clinical and research tool for the assessment of daytime sleepiness in obstructive sleep apnea. ${ }^{10,17,18}$

\section{FOSQ}

The FOSQ is a sleep-specific self-report questionnaire that has been used in research and clinical practice to measure the impact of daytime sleepiness on the activities of daily living. ${ }^{11}$ The FOSQ is a self-administered, sleep-specific self-report questionnaire that contains 30 items. There are five factorbased subscales: activity level, vigilance, intimacy and sexual relationships, general productivity and social outcomes. A weighted mean item score is calculated for each subscale, and the sum of the subscales produces a global score. ${ }^{11}$ This questionnaire is designed to assess the impact of disorders of excessive sleepiness on multiple activities of everyday living with internal validity and test-retest reliability. ${ }^{11}$ 


\section{ODSI}

The ODSI is a three-item questionnaire that is administered as an interview by a health professional. Each item is rated on a 7-point Likert scale, and the total score ranges from 0 to 24 . The first item scored from 0 to 12 helps to assess sleepiness or falling asleep in active situations or situations where a high level of stimulation is required $(0=$ not at all, $8=$ mildly, $10=$ moderately and $12=$ very frequently). As falling asleep during active situations (the first item) is likely more dangerous, Onen et $\mathrm{al}^{13}$ proposed to score it higher than following items. The second item scored from 0 to 6 helps to assess sleepiness during passive situations, such as while reading or watching TV $(0=$ not at all, $2=$ mildly, $4=$ moderately and $6=$ very frequently). The third item helps to estimate the average total duration of sleep, including sleepiness, falling asleep and naps, during the day $(0=\sim £ 0.5$ hours, $2=\sim 2$ hours, $4=\sim 4$ hours and $6=\sim \geq 6$ hours). The rater decides whether the rating lies on the defined scale steps $(0,2,4,6)$ or between them $(1,3,5)$. The intention of the ODSI is to derive a measure of sleepiness by combining all items on the short questionnaire. The validation study of this questionnaire demonstrated that these items would ensure good coverage in the target population with high inter-rater agreement and test-retest reliability. ${ }^{13}$

\section{Data analysis}

Spearman's rank correlations were run to measure the strength and direction of the linear relationship between the combined total scores of the ODSI and both the combined scores of the ESS and global FOSQ. Correlations between the subscales and the ODSI were also tested using Spearman's $\rho$. In addition, we tested the response rates among all questionnaires via the test of proportions. Generalized linear models were also run to compare each item in the ODSI to that in the ESS and FOSQ. As complementary analyses, we also explored correlations between the three questionnaires and apnea severity (using Apnea-Hypopnea Index [AHI]) via Spearman's $\rho$.

\section{Ethical approval}

All procedures performed in studies involving human participants were in accordance with the ethical standards of the institutional and/or national research committee and the 1964 Declaration of Helsinki and its later amendments or comparable ethical standards.

\section{Informed consent}

Written informed consent was obtained from all individual participants included in the study.

\section{Results}

\section{Descriptive data}

There were a total of 125 subjects in this study. The mean \pm standard deviation (SD) age was $70.9 \pm 5.3$ years, and subjects were predominantly male $(58.9 \%)$ of Caucasian ethnicity $(77.2 \%)$ and had a mean body mass index (BMI) of $33.0 \pm$ $6.9 \mathrm{~kg} / \mathrm{m}^{2}$ and moderate sleep apnea with an AHI of $31.9 \pm$ 27.9 events/hour. Subjects had average scores of $7.67 \pm 5.92$ on the ODSI, $8.00 \pm 4.65$ on the ESS and 17.6 \pm 2.2 on the FOSQ. Table 1 lists the main sample characteristics of these subjects as well as questionnaire scores for the ODSI, ESS and FOSQ, and Table 2 demonstrates the sample demographics categorized by ESS $\geq 10$ and $\mathrm{ESS}<10$.

\section{Comparison of ODSI with FOSQ and ESS}

Figures 1 and 2 show scatter plots of total combined ODSI versus ESS and total FOSQ scores, respectively. A positive association was indicated on a scatter plot by an upward trend where higher ODSI scores corresponded with higher ESS scores. A negative association between ODSI and FOSQ score was seen where the higher ODSI score was associated with a lower FOSQ score. This was expected, as a higher FOSQ score is associated with a more positive outcome, while in the ODSI, a higher score is associated with a more negative outcome. Correlations of ODSI versus ESS and

Table I Baseline demographic and questionnaire results

\begin{tabular}{lll}
\hline Characteristic & Number & $\begin{array}{l}\text { Mean } \pm \text { SD (continuous)/ } \\
\text { Percentages (categorical) }\end{array}$ \\
\hline $\begin{array}{l}\text { Age, years } \\
\text { Gender }\end{array}$ & 125 & $70.9 \pm 5.27$ \\
Male & 73 & $58.9 \%$ \\
Female & 51 & $41.1 \%$ \\
Race and ethnicity & & \\
African American & 27 & $22.0 \%$ \\
Asian & 1 & $0.81 \%$ \\
Caucasian & 95 & $77.2 \%$ \\
BMI, kg/m ${ }^{2}$ & 125 & $33.0 \pm 6.92$ \\
AHI, events/hour & 124 & $31.9 \pm 27.9$ \\
Questionnaires & & \\
ODSI & 122 & $7.67 \pm 5.92$ \\
ESS & 115 & $8.00 \pm 4.65$ \\
FOSQ & 124 & $17.6 \pm 2.15$ \\
\hline
\end{tabular}

Notes: a One subject was missing gender information. ${ }^{\mathrm{b}} \mathrm{T}$ wo subjects were missing race information. 'Continuous characteristics are presented as mean \pm SD and categorical covariates as percentages. All subjects included in analyses had normal cognitive function (MMSE score $>24$ ) and age $\geq 65$ and represented questionnaires with no missing data.

Abbreviations: BMI, body mass index; AHI, Apnea-Hypopnea Index; ODSI, Observation and Interview-based Diurnal Sleepiness Inventory; ESS, Epworth Sleepiness Score; FOSQ, Functional Outcomes of Sleep Questionnaire; MMSE, MiniMental State Examination. 
FOSQ were then tested using Spearman's $\rho$. The Spearman's rank correlation analysis confirmed that the questionnaire items of the ODSI, designed to assess the sleepiness, correlated significantly with the total ESS score (Spearman's $\rho: 0.67, P=0.000$ ) with 112 subjects filling out both ques-

Table 2 Summary statistics of patient demographics by sleepiness status (ESS $\geq 10$, sleepy; ESS $<10$, non-sleepy)

\begin{tabular}{lll}
\hline Characteristic & \multicolumn{2}{l}{ Sleepiness } \\
\cline { 2 - 3 } & No (n= 77) & Yes (n= 35) \\
\hline $\begin{array}{l}\text { Age, years } \\
\text { Gender }\end{array}$ & $71.4(1 \mathrm{I} . \mathrm{I})$ & $69.4(4.46)$ \\
$\quad$ Female & $27(35.5)$ & $020(57.1 \%)$ \\
$\quad$ Male & $49(64.5)$ & $015(42.9 \%)$ \\
Race/ethnicity & & \\
$\quad$ African American & $16(21.3 \%)$ & $9(25.71 \%)$ \\
$\quad$ Asian & 0 & $1(2.86 \%)$ \\
$\quad$ Caucasian & $59(78.7 \%)$ & $25(71.4 \%)$ \\
BMI, kg/m & $33.2(7.07)$ & $33.7(7.26)$ \\
AHI, events/hour & $30.8(25.5)$ & $33.2(34.0)$ \\
Questionnaires & & \\
ODSI & $5.18(4.62)$ & $13.0(5.0)$ \\
ESS & $5.23(2.31)$ & $13.6(2.7)$ \\
FOSQ & $18.2(1.7)$ & $16.35(2.32)$ \\
\hline
\end{tabular}

Notes: a ${ }^{\circ}$ en subject was missing gender information. ${ }^{\mathrm{b}} \mathrm{T}$ wo subjects were missing race information. 'Continuous characteristics are presented as mean \pm SD and categorical covariates as percentages. All subjects included in analyses had normal cognitive function (MMSE score $>24$ ) and age $\geq 65$ and represented questionnaires with no missing data. Abbreviations: ESS, Epworth Sleepiness Score; BMI, body mass index; AHI, Apnea-Hypopnea Index; ODSI, Observation and Interview-based Diurnal Sleepiness Inventory; FOSQ, Functional Outcomes of Sleep Questionnaire; MMSE, Mini-Mental State Examination. tionnaires. The ODSI and FOSQ were negatively correlated (Spearman's $\rho$ : $-0.52, P=0.000$ ) with 121 subjects filling out both questionnaires (Table 3 ).

\section{Associations between individual ODSI items and ESS and FOSQ}

We then conducted analyses of possible associations between ODSI questionnaire items and combined ESS and FOSQ global scores by using general linear models that were specific to the individual ODSI item. The ODSI 1 item (assessing sleepiness in active situations) was correlated with the ESS combined score $(\beta=0.14 ; 95 \% \mathrm{CI},-0.01$ to $0.29 ; P=0.069)$. ODSI 2 item (sleepiness in passive situations) was significantly correlated with the ESS ( $\beta=1.65 ; 95 \% \mathrm{CI}, 1.32$ to 1.98; $P<0.001)$. Both ODSI 1 and ODSI 2 were correlated with FOSQ: ODSI $1, \beta=-0.15,95 \% \mathrm{CI},-0.24$ to $-0.07, P$ $<0.001$ and ODSI $2, \beta=-0.35,95 \% \mathrm{CI},-0.55$ to $0.16, P$ $<0.001)$. ODSI 3 item was not significantly correlated as expected, as this is a question about estimating average total duration of sleep during the day.

\section{Associations of ODSI to subscales of FOSQ}

As the FOSQ determines how sleepiness affects a person's actual daily ability to function, ${ }^{11}$ we explored how well the ODSI captures this measurement by comparing the ODSI to

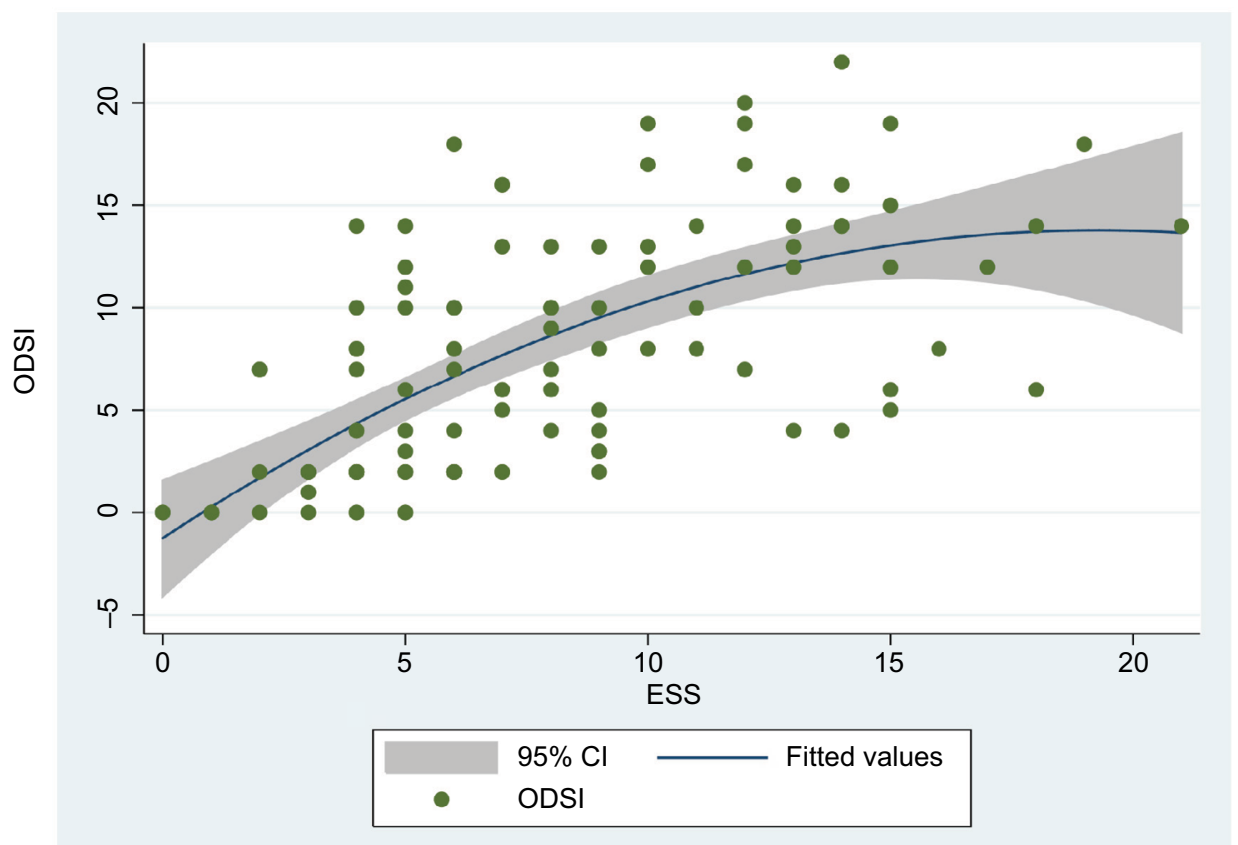

Figure I Scatter plot of ODSI versus ESS.

Abbreviations: ODSI, Observation and Interview-based Diurnal Sleepiness Inventory; ESS, Epworth Sleepiness Scale; Cl, confidence interval. 


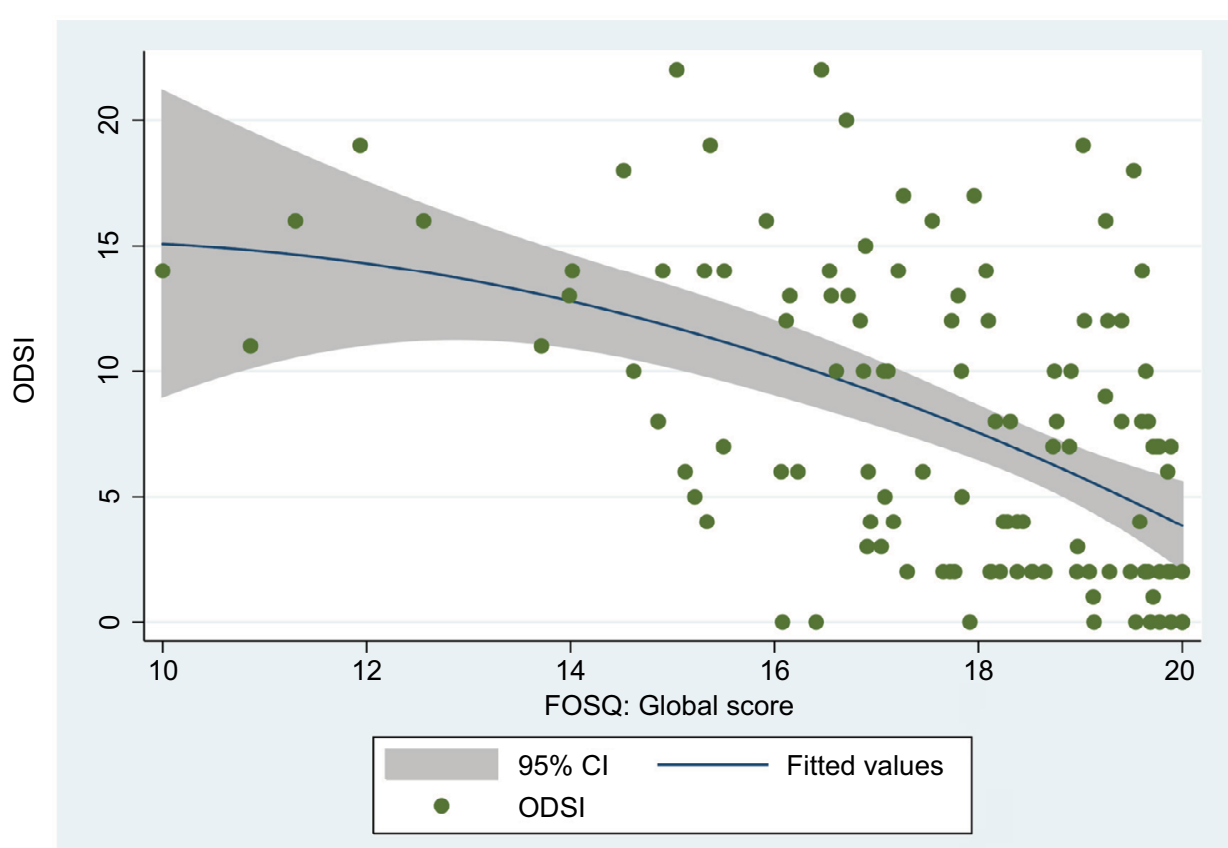

Figure 2 Scatter plot of ODSI versus FOSQ.

Abbreviations: ODSI, Observation and Interview-based Diurnal Sleepiness Inventory; FOSQ, Functional Outcomes of Sleep Questionnaire; CI, confidence interval.

Table 3 Spearman's $\rho$ nonparametric correlation coefficient between ODSI and ESS and ODSI and FOSQ global questionnaires, respectively

\begin{tabular}{llll}
\hline $\begin{array}{l}\text { Questionnaire } \\
\text { comparison }\end{array}$ & Number & Spearman's $\rho$ & $P$-value \\
\hline ODSI and ESS & 112 & 0.67 & $<0.00 \mathrm{I}$ \\
ODSI and FOSQ & 121 & $-0.52^{\mathrm{a}}$ & $<0.00 \mathrm{I}$ \\
\hline
\end{tabular}

Note: a There is a negative correlation as a higher FOSQ score is associated with a more positive outcome, while in the ODSI, a higher score is associated with a more negative outcome.

Abbreviations: ODSI, Observation and Interview-based Diurnal Sleepiness Inventory; ESS, Epworth Sleepiness Score; FOSQ, Functional Outcomes of Sleep Questionnaire.

each FOSQ subscale. The five subscales included activity level, vigilance, intimacy and sexual relationships, general productivity and social outcome. ${ }^{11}$ Correlations between the subscales and the ODSI were tested using Spearman's $\rho$. The Spearman's rank correlation analysis confirmed that the ODSI correlated significantly with the FOSQ under all subscales (Table 4).

\section{Response rates}

The response rates were calculated by the total number of subjects who were administered the questionnaire and completed the questionnaires in full with no missing data. The response rate for the ODSI was $97.6 \%$ compared to $91.7 \%$ for the ESS. There were significant differences in response rates between the ODSI and the ESS (test of proportions; $P=0.009$ ). The FOSQ response rate was not calculated as the scoring of the
Table 4 Spearman's $\rho$ nonparametric correlation coefficient between ODSI and five subscales of the FOSQ

\begin{tabular}{llll}
\hline Subscale & Number & Spearman's $\rho$ & $\boldsymbol{P}$-value \\
\hline Activity level & $12 \mathrm{I}$ & $-0.5 \mathrm{I}$ & $<0.00 \mathrm{I}$ \\
Vigilance & $12 \mathrm{I}$ & -0.44 & $<0.00 \mathrm{I}$ \\
Sexual & 75 & -0.33 & 0.0043 \\
Social & 120 & $-0.3 \mathrm{I}$ & 0.0005 \\
General productivity & 120 & -0.33 & 0.0002 \\
\hline
\end{tabular}

Abbreviations: ODSI, Observation and Interview-based Diurnal Sleepiness Inventory; FOSQ, Functional Outcomes of Sleep Questionnaire.

FOSQ suggests that a response score of 0 for an item should be counted as not applicable (N/A) or missing response, and the mean of the answered items (weighted mean item total) with responses $\geq 1$ for each subscale was used. ${ }^{11}$

\section{Correlation of ODSI to AHI}

As a complementary analysis, we tested the associations of the ODSI, ESS and FOSQ as they relate to continuous AHI. There were no correlations between the ODSI and the AHI (Spearman's $\rho$ : $-0004, P=1.00)$. Similarly both the ESS (Spearman's $\rho$ : $-0.04,0.70$ ) and the FOSQ (Spearman's $\rho$ : $0.12, P=0.19$ ) did not show any significant correlations with AHI (data not shown).

\section{Discussion}

The findings of our study demonstrate that the ODSI is efficient in that it is a simple three-question questionnaire taking 
less time to complete and is a suitable new measurement of sleepiness among older adults that will aid in improving the quality of care. The ODSI demonstrated the ability to capture sleepiness measures in this population, and reporting bias is reduced by the general questions within the ODSI.

AHI was not significantly correlated with any of the subjective measures of sleepiness as seen in prior literature. ${ }^{19,20}$ This finding supports the research that suggests factors other than respiratory events and arousal lead to sleepiness. For instance, the average AHI of our population was moderate, and sleep-disordered breathing in older adults has been suggested as representing a different process relative to sleep-disordered breathing in younger subjects. ${ }^{21}$ Specifically, age-dependent sleep apnea is likely to develop with advancing age and may be secondary to age-dependent conditions such as increasing weight and increased airway collapsibility. ${ }^{22,23}$ As sleep apnea is likely to develop with advancing age, it is important to consider this interaction when understanding the mechanisms of sleepiness among older adults. Multiple other factors should also be considered (i.e., inflammatory cytokines, comorbid conditions that may increase sympathetic nervous activity and reduce sleepiness) when exploring sleepiness in the elderly population. The strengths of our study include the large sample size allowing for the calculation of response rates and agreement between questionnaires. The ODSI is short and simple to complete, and the general nature of the ODSI questions eliminates potential issue of older adults not being able to answer all items. The act of asking whether patients felt sleepy in active or passive situations eliminated any potential for the issue of most older adults not able to answer all of ESS items, ${ }^{12}$ due to the irrelevance of the question (i.e., feeling sleepy while operating a motor vehicle) or potential response bias for sensitive questions. The ODSI is a useful alternative for capturing sleepiness, particularly among older adults, as the general questions allow for a broader range of applicability and thus may be useful for other subject populations as well.

The limitations of our study include a limited amount of data obtained from polysomnography. The structure of the ODSI is subject to interviewer bias, when an interviewer influences responses from participants, subtly communicating expected answers. However, this bias is reduced as the questions in the ODSI are prepared directly from the structured questionnaire with designated response items available. Furthermore, response rates were significantly higher in the ODSI compared to the ESS in our population. The ODSI is a simple screening tool designed to improve the accuracy of ascertaining sleepy versus non-sleepy subjects among older adults, which will facilitate identification of treatable sleep disorders among this population. Future research should explore the ability of the ODSI to assess sleepiness among other populations. Owing to the ease of completion and high agreement with current measures for sleepiness, the ODSI can be integrated into routine clinical assessment among older adults.

\section{Acknowledgments}

The National Institutes of Health (NIH) provided financial support in the form of 1K99NR014675-01/R00NR01467503 funding (Pak). The sponsor had no role in the design or conduct of this research. The authors acknowledge Swathi Yarlagadda, M.B.B.S. for her help with literature review.

\section{Disclosure}

All authors certify that they have no affiliations with or involvement in any organization or entity with any financial interest (such as honoraria; educational grants; participation in speakers' bureaus; membership, employment, consultancies, stock ownership or other equity interest and expert testimony or patent-licensing arrangements) or nonfinancial interest (such as personal or professional relationships, affiliations, knowledge or beliefs) in the subject matter or materials discussed in this manuscript. The authors report no conflicts of interest in this work.

\section{References}

1. Mant A, Eyland EA. Sleep patterns and problems in elderly general practice attenders: an Australian survey. Comm Health Stud. 1988;12(2):192-199.

2. Asplund R. Daytime sleepiness and napping amongst the elderly in relation to somatic health and medical treatment. J Intern Med. 1996;239(3):261-267.

3. Whitney CW, Enright PL, Newman AB, Bonekat W, Foley D, Quan SF. Correlates of daytime sleepiness in 4578 elderly persons: the Cardiovascular Health Study. Sleep. 1998;21(1):27-36.

4. Ganguli M, Reynolds CF, Gilby JE. Prevalence and persistence of sleep complaints in a rural older community sample: the MoVIES project. J Am Geriatr Soc. 1996;44(7):778-784.

5. Onen F, Higgins S, Onen SH. Falling-asleep-related injured falls in the elderly. J Am Med Dir Assoc. 2009;10(3):207-210.

6. Merlino G, Piani A, Gigli GL, et al. Daytime sleepiness is associated with dementia and cognitive decline in older Italian adults: a populationbased study. Sleep Med. 2010;11(4):372-377.

7. Empana JP, Dauvilliers Y, Dartigues JF, et al. Excessive daytime sleepiness is an independent risk indicator for cardiovascular mortality in community-dwelling elderly: the three city study. Stroke. 2009;40(4):1219-1224.

8. Newman AB, Spiekerman CF, Enright P, et al. Daytime sleepiness predicts mortality and cardiovascular disease in older adults. The Cardiovascular Health Study Research Group. JAm Geriatr Soc. 2000;48(2): 115-123.

9. Ruggles K, Hausman N. Evaluation of excessive daytime sleepiness. WMJ. 2003;102(1):21-24.

10. Johns MW. A new method for measuring daytime sleepiness: the Epworth Sleepiness Scale. Sleep. 1991;14(6):540-545. 
11. Weaver TE, Laizner AM, Evans LK, et al. An instrument to measure functional status outcomes for disorders of excessive sleepiness. Sleep. 1997;20(10):835-843.

12. Onen F, Moreau T, Gooneratne NS, Petit C, Falissard B, Onen SH. Limits of the Epworth Sleepiness Scale in older adults. Sleep Breath. 2013;17(1):343-350.

13. Onen F, Lalanne C, Pak VM, Gooneratne N, Falissard B, Onen SH. A 3-item instrument for measuring daytime sleepiness: the observation and interview based diurnal sleepiness inventory (ODSI). J Clin Sleep Med. 2016;12(4):505-512.

14. Kumru H, Santamaria J, Belcher R. Variability in the Epworth Sleepiness Scale score between the patient and the partner. Sleep Med. 2004;5(4):369-371.

15. Faria AC, da Costa CH, Rufino R. Sleep apnea clinical score, Berlin questionnaire, or Epworth Sleepiness Scale: which is the best obstructive sleep apnea predictor in patients with COPD? Int J Gen Med. 2015;8:275-281.

16. Tombaugh TN, McIntyre NJ. The mini-mental state examination: a comprehensive review. J Am Geriatr Soc. 1992;40(9):922-935.
17. Johns MW. Sleepiness in different situations measured by the Epworth Sleepiness Scale. Sleep. 1994;17(8):703-710.

18. Johns MW. Reliability and factor analysis of the Epworth Sleepiness Scale. Sleep. 1992;15(4):376-381.

19. Macey PM, Woo MA, Kumar R, Cross RL, Harper RM. Relationship between obstructive sleep apnea severity and sleep, depression and anxiety symptoms in newly-diagnosed patients. PLoS One. 2010;5(4):e10211.

20. Bausmer U, Gouveris H, Selivanova O, Goepel B, Mann W. Correlation of the Epworth Sleepiness Scale with respiratory sleep parameters in patients with sleep-related breathing disorders and upper airway pathology. Eur Arch Otorhinolaryngol. 2010;267(10):1645-1648.

21. Berry DT, Phillips BA, Cook YR, et al. Geriatric sleep apnea syndrome: a preliminary description. J Gerontol. 1990;45(5):M169-M174.

22. Bliwise DL. Sleep apnea and cognitive function: where do we stand now? Sleep. 1993;16(8 Suppl):S72-S73.

23. Bliwise DL. Sleep in normal aging and dementia. Sleep. 1993;16(1):40-81.
Nature and Science of Sleep

\section{Publish your work in this journal}

Nature and Science of Sleep is an international, peer-reviewed, open access journal covering all aspects of sleep science and sleep medicine, including the neurophysiology and functions of sleep, the genetics of sleep, sleep and society, biological rhythms, dreaming, sleep disorders and therapy, and strategies to optimize healthy sleep. The manuscript

\section{Dovepress}

management system is completely online and includes a very quick and fair peer-review system, which is all easy to use. Visit http://www. dovepress.com/testimonials.php to read real quotes from published authors. 